\title{
Interpolating Between Periodicity and Discreteness Through the Fractional Fourier Transform
}

\author{
Haldun M. Ozaktas and Uygar Sümbül, Student Member, IEEE
}

\begin{abstract}
Periodicity and discreteness are Fourier duals in the same sense as operators such as coordinate multiplication and differentiation, and translation and phase shift. The fractional Fourier transform allows interpolation between such operators which gradually evolve from one member of the dual pair to the other as the fractional order goes from zero to one. Here, we similarly discuss the interpolation between the dual properties of periodicity and discreteness, showing how one evolves into the other as the order goes from zero to one. We also discuss the concepts of partial discreteness and partial periodicity and relate them to fractional discreteness and periodicity.
\end{abstract}

Index Terms-Chirp functions, discrete functions, fractional Fourier transform, periodic functions, sampling.

\section{INTRODUCTION}

$\mathbf{P}$ ERIODICITY and discreteness are Fourier duals (or Fourier conjugates) in the sense that the Fourier transform of a periodic function is discrete and the Fourier transform of a discrete function is periodic. A periodic function $f_{\text {per }}(u)$ can be defined as one obtained by periodically replicating an arbitrary function $f(u)$

$$
f_{\mathrm{per}}(u)=\sum_{k=-\infty}^{\infty} f(u-k \Delta u)
$$

for a specific $\Delta u>0$, referred to as the period. A discrete function $f_{\text {dis }}(u)$ can be defined as one obtained by uniformly sampling an arbitrary function $f(u)$

$$
f_{\text {dis }}(u)=\delta u \sum_{k=-\infty}^{\infty} f(k \delta u) \delta(u-k \delta u)
$$

for a specific $\delta u>0$, referred to as the sampling interval. Discrete functions, as defined, may also be referred to as impulsive functions. The reason for including the factor $\delta u$ in front of the summation will become apparent in Section IV.

Several common pairs of operators are Fourier duals (or conjugates) in the same sense. Coordinate multiplication and differentiation, translation and phase shift, and chirp multiplication and chirp convolution are well-known examples. The scaling

Manuscript received March 16, 2005; revised November 23, 2005. The associate editor coordinating the review of this manuscript and approving it for publication was Prof. Karim Drouiche. The work of H. M. Ozaktas is supported in part by the Turkish Academy of Sciences.

H. M. Ozaktas is with the Department of Electrical Engineering, Bilkent University, TR-06800 Bilkent, Ankara, Turkey (e-mail: haldun@ee.bilkent.edu.tr).

U. Sümbül is with the Department of Electrical Engineering, Stanford University, Stanford, CA 94305-9505 USA (e-mail: uygar@ stanford.edu).

Digital Object Identifier 10.1109/TSP.2006.880224 operation is its own dual with reciprocal parameter. (For a discussion of such operators and duality, see, for instance, [1]-[4].)

The fractional Fourier transform operator $\mathcal{F}^{a}$ is a generalization of the ordinary Fourier transform operator $\mathcal{F}$. Although it can be defined in a number of equivalent ways [1], a common approach is to define it as the ath operator power of the ordinary Fourier operator, as already built into the notation. The fractional Fourier transform interpolates between the identity operator $\mathcal{F}^{0}=\mathcal{I}$ and the ordinary Fourier operator $\mathcal{F}^{1}=\mathcal{F}$ in a continuous manner. Based on the fractional Fourier transform, it is possible to interpolate between the above mentioned common dual pairs of operators (see [1] and [4] and the references therein). These interpolations gradually evolve from one member of the dual pair to the other as the fractional order goes from zero to one. These results will be reviewed in Section III.

In this paper we discuss the interpolation between the dual properties of periodicity and discreteness. We define the concepts of fractional periodicity and fractional discreteness with order parameter $a$, showing how one evolves into the other as the order goes from zero to one. Although the duality between periodicity and discreteness is widely understood, the interpolation between these two properties does not seem to have received any attention.

We also introduce the concepts of partial periodicity and discreteness. In real life, ideal delta sampled functions and infinite periodic functions do not exist. The sampling pulse is finite, reflecting a finite resolution, and only a finite number of periods can exist. The concepts of partial periodicity and discreteness are important because they model such real-life sampled and periodic functions. An important result we derive is that relating these partial operators to fractional periodization and discretization operators. Therefore, under certain idealizations, the fractional periodization and discretization operators also model real-life sampled and periodic functions.

The study of periodic functions is closely related to the study of functions of finite extent. A periodic function may be considered a redundant representation of a function of finite extent; alternatively a function of finite extent may be considered a compact representation of a periodic function (with the period or finite extent being specified in both cases). Therefore, the discussion of the present paper is relevant for functions of finite extent as well.

While a function $f(u)$ and its Fourier transform $F(\mu)=$ $\int_{-\infty}^{\infty} f(u) \exp (-i 2 \pi \mu u) d u$ cannot both be of finite extent in the strict mathematical sense, in practice we typically assume that both are at least approximately of finite extent in the sense that the signal energy falling outside of that extent is negligible. Let the extent of $f(u)$ be denoted by $\Delta u$ and the extent of $F(\mu)$ 
be denoted by $\Delta \mu$, both assumed to cover an interval symmetric around the origin. The sampling theorem implies that a sampling interval of $\delta u=1 / \Delta \mu$ and $\delta \mu=1 / \Delta u$ will be sufficient in the $u$ and $\mu$ domains respectively. In other words, the extent of the signal (or its period) in one domain determines its resolution in the other domain.

This implies that $\Delta u \Delta \mu$ samples will be sufficient to completely characterize the function in both domains. This number, which we will denote by $N$, is referred to as the time-bandwidth product of the signal, or its number of degrees of freedom. To the extent that the energy outside $\Delta u$ and $\Delta \mu$ is negligible, the discrete Fourier transform (DFT) maps the $N$ time samples to the $N$ frequency samples. The exact relationship is given by a form of Poisson's formula [5]

$$
F_{\text {per }}(m / \Delta u)=\frac{1}{\Delta \mu} \sum_{n=0}^{N-1} f_{\text {per }}(n / \Delta \mu) \exp (-i 2 \pi m n / N)
$$

which is a DFT between periodically replicated versions of $f(u)$ and $F(\mu)$. Here, $0 \leq m \leq N-1$ is an integer, $\Delta u$ and $\Delta \mu$ are arbitrary, and $F_{\text {per }}(\mu)=\sum_{k=-\infty}^{\infty} F(\mu-k \Delta \mu)$ and $f_{\text {per }}(u)=$ $\sum_{k=-\infty}^{\infty} f(u-k \Delta u)$. Further discussion of these issues may be found in [1, sec. 3.3]. General references on sampling and interpolation include [5]-[7].

In the next section, we define the fractional Fourier transform and its relation to the Wigner distribution. In Section III, we discuss several pairs of dual operators and consolidate their fractional generalizations, which are continuums of operators indexed by the fractional-order parameter $a$. Section IV defines the discretization and periodization operators and their fractional generalizations. Finally, partial versions of discretization and periodization operators are discussed in Section V.

Throughout the paper, $\sqrt{z}$ will mean the square root of $z$ whose argument lies in the interval $(-\pi / 2, \pi / 2]$ and we assume that $f(u)$ belongs to the Schwarz space of rapidly decreasing functions; these are the infinitely differentiable functions whose derivatives of all orders (including the function itself) have faster than polynomial decay. This space is dense in $L^{2}(\mathbf{R})$. In other words, any square-integrable function can be approximated to arbitrary accuracy by a rapidly decreasing function.

\section{FRACTIONAL FOURIER TRANSFORM}

The fractional Fourier transform has received considerable interest over the past ten years and has found many applications [8]-[23]. An important concept is that of fractional Fourier domains, which are generalizations of the ordinary time and frequency domains [24], [25]. This continuum of domains provides a continuous transition between the time and frequency domains.

The ath-order fractional Fourier transform $f_{a}(u)=$ $\left\{\mathcal{F}^{a} f\right\}(u)$ of the function $f(u)$ is defined for $0<|a|<2$ as

$$
\begin{aligned}
\mathcal{F}^{a}[f(u)] \equiv & \left\{\mathcal{F}^{a} f\right\}(u) \equiv \int_{-\infty}^{\infty} K_{a}\left(u, u^{\prime}\right) f\left(u^{\prime}\right) d u^{\prime} \\
K_{a}\left(u, u^{\prime}\right) \equiv & \frac{\exp [-i(\pi \operatorname{sgn}(\alpha) / 4-\alpha / 2)]}{|\sin \alpha|^{1 / 2}} \\
& \times \exp \left[i \pi\left(u^{2} \cot \alpha-2 \csc \alpha u u^{\prime}+u^{\prime 2} \cot \alpha\right)\right]
\end{aligned}
$$

where

$$
\alpha \equiv \frac{a \pi}{2}
$$

The definition may be extended outside the interval $(-2,2)$ through $\mathcal{F}^{4 j+a} f=\mathcal{F}^{a} f$ for any integer $j$. Moreover, $\mathcal{F}^{4 j}$, $\mathcal{F}^{4 j+1}$, and $\mathcal{F}^{4 j+2}$ correspond to the identity operator $\mathcal{I}$, the ordinary Fourier transform operator $\mathcal{F}$, and the parity operator $\mathcal{P}=\mathcal{F}^{2}$, respectively. The transform is a linear operator and it is additive in index: $\mathcal{F}^{a_{1}} \mathcal{F}^{a_{2}} f=\mathcal{F}^{a_{1}+a_{2}} f$. We also note that as a result of index additivity, it is actually sufficient to define the transform for $0 \leq a<1$, since it can then be easily generalized to other values of $a$ using $\mathcal{F}^{a}=\mathcal{F}^{a-1} \mathcal{F}$ for $1<a<2$, and $\mathcal{F}^{a}=\left(\mathcal{F}^{-a}\right)^{-1}$ for $-2<a<0$. Other properties are given in [1].

The Wigner distribution $W_{f}(u, \mu)$ of $f(u)$ is defined as

$$
\begin{aligned}
& W_{f}(u, \mu) \\
& =\int_{-\infty}^{\infty} f\left(u+u^{\prime} / 2\right) f^{*}\left(u-u^{\prime} / 2\right) \exp \left(-i 2 \pi \mu u^{\prime}\right) d u^{\prime} \\
& =\int_{-\infty}^{\infty} F\left(\mu+\mu^{\prime} / 2\right) F^{*}\left(\mu-\mu^{\prime} / 2\right) \exp \left(i 2 \pi \mu^{\prime} u\right) d \mu^{\prime}
\end{aligned}
$$

where $F(\mu)$ is the Fourier transform of $f(u)$ and * indicates complex conjugation. Properties of the Wigner distribution are discussed in many sources, for instance see [1], [26], [27].

The effect of $a$ th-order fractional Fourier transformation on the Wigner distribution of a signal is to rotate it by an angle $\alpha$ [28]. Hence, the mathematical relation between the Wigner distribution of a function and that of its fractional Fourier transform is as follows:

$$
W_{f_{a}}(u, \mu)=W_{f}(u \cos \alpha-\mu \sin \alpha, u \sin \alpha+\mu \cos \alpha) .
$$

The Radon transform operator $\mathcal{R} \mathcal{D} \mathcal{N}_{\alpha}$, which takes the integral projection of the function $W_{f}(u, \mu)$ onto an axis making angle $\alpha=a \pi / 2$ with the $u$ axis, can be used to restate the previous property in the following manner:

$$
\left\{\mathcal{R D N}_{\alpha}\left[W_{f}(u, \mu)\right]\right\}\left(u_{a}\right)=\left|f_{a}\left(u_{a}\right)\right|^{2}
$$

Here, the projection axis $u_{a}$ making angle $\alpha$ with the $u$ axis, is referred to as the $a$ th fractional Fourier domain [24], [25]. The time and frequency domains are merely special cases of the continuum of fractional Fourier domains. Throughout this paper, $u, \mu$, and more generally $u_{a}$, will be treated as dimensionless variables.

The fractional Fourier transforms of $\delta(u-\xi)$ and $\exp (i 2 \pi \eta u)$ are given, respectively, by [1]

$$
\begin{aligned}
& \sqrt{1-i \cot \alpha} \exp \left[i \pi\left(u^{2} \cot \alpha-2 u \xi \csc \alpha+\xi^{2} \cot \alpha\right)\right] \\
& \sqrt{1+i \tan \alpha} \exp \left[-i \pi\left(u^{2} \tan \alpha-2 u \eta \sec \alpha+\eta^{2} \tan \alpha\right)\right]
\end{aligned}
$$

When $a=0$, the first expression reduces to $\delta(u-\xi)$ and the second expression reduces to $\exp (i 2 \pi \eta u)$. When $a=1$, the first expression reduces to $\exp (-i 2 \pi \xi u)$ and the second expression reduces to $\delta(u-\eta)$. As is well known, the delta function and 
harmonic function are Fourier duals of each other. For intermediate values of $a$, we see that the result is in general a chirp function which interpolates between a delta function and a harmonic function as $a$ varies from zero to one. It also follows that the fractional Fourier transform of a sampled function or a periodic function are both weighted summations of chirps.

\section{COMMON DUAL OPERATORS AND THEIR FRACTIONAL GENERALIZATIONS}

The dual of the operator $\mathcal{A}$ will be denoted by $\mathcal{A}^{\mathrm{D}}$ and satisfies

$$
\begin{aligned}
\mathcal{A}^{\mathrm{D}} & =\mathcal{F}^{-1} \mathcal{A} \mathcal{F}, \\
\mathcal{A} & =\mathcal{F} \mathcal{A}^{\mathrm{D}} \mathcal{F}^{-1} .
\end{aligned}
$$

$\mathcal{A}^{\mathrm{D}}$ performs the same action on the frequency-domain representation $F(\mu)$, that $\mathcal{A}$ performs on the time-domain representation $f(u)$.

The "fractional" operators we deal with in this paper perform the same action in a fractional domain, that their ordinary counterpart performs in the time domain. More explicitly, the fractional operator has the same effect on the $a$ th fractional Fourier transform $f_{a}\left(u_{a}\right)$ in the $a$ th domain, as the original operator has on the original function $f(u)$ in the time domain. Mathematically, this can be expressed as

$$
\mathcal{A}_{a}=\mathcal{F}^{-a} \mathcal{A F}^{a} .
$$

This equation constitutes a generalization of (10) and reduces to it when $a=1$ with $\mathcal{A}_{1}=\mathcal{A}^{\mathrm{D}}$. Note that $\mathcal{A}$ and $\mathcal{A}_{a}$ are two different operators, whose representations in the zeroth and $a$ th domains, respectively, are identical. (The zeroth domain is the time domain.) They are not different representations of the same operator. Also note that, to distinguish such "fractional" operators associated with the $a$ th fractional Fourier domain, from the ath operator power of $\mathcal{A}$ which is denoted by $\mathcal{A}^{a}$, we are denoting them by $\mathcal{A}_{a}$. To further discriminate the two cases, we note that for $a=0, \mathcal{A}_{0}=\mathcal{A}$ while $\mathcal{A}^{0}=\mathcal{I}$; and for $a=1$, $\mathcal{A}_{1}=\mathcal{A}^{\mathrm{D}}$ while $\mathcal{A}^{1}=\mathcal{A}$. In yet other words, $\mathcal{A}_{a}$ interpolates between the operator $\mathcal{A}$ and its dual $\mathcal{A}^{\mathrm{D}}$, whereas $\mathcal{A}^{a}$ interpolates between the identity operator and the operator $\mathcal{A}$. Equation (11) also holds for $\mathcal{A}^{\mathrm{D}}$, as follows:

$$
\mathcal{A}_{a}^{\mathrm{D}}=\mathcal{F}^{-a} \mathcal{A}^{\mathrm{D}} \mathcal{F}^{a} .
$$

Since $\left(\mathcal{A}^{\mathrm{D}}\right)_{a}=\left(\mathcal{A}_{a}\right)^{\mathrm{D}}$ as can be easily shown from (11), we denote both simply by $\mathcal{A}_{a}^{\mathrm{D}}$.

In what follows, we summarily discuss several common dual pairs of operators and their fractional versions. A more detailed treatment can be found in [4]. The symbols used to denote operators are inherited from [1] and [4].

We begin by defining the coordinate multiplication operator $\mathcal{U}$ and the differentiation operator $\mathcal{D}$ through their effects in the time domain, as follows:

$$
\begin{aligned}
& \{\mathcal{U} f\}(u)=u f(u), \\
& \{\mathcal{D} f\}(u)=(i 2 \pi)^{-1} f^{\prime}(u) .
\end{aligned}
$$

It can be easily shown that these operators constitute a dual pair with $\mathcal{A}=\mathcal{U}$ and $\mathcal{A}^{\mathrm{D}}=\mathcal{D}$ and satisfy (10). Likewise, it can be shown that $\mathcal{D}$ and $-\mathcal{U}$ are also a dual pair. Note that $\mathcal{D}$ indeed has the same effect of coordinate multiplication in the frequency domain, as $\mathcal{U}$ has in the time domain. (This follows from the property that the Fourier transform of $(i 2 \pi)^{-1} f^{\prime}(u)$ is $\mu F(\mu)$.)

The fractional forms of these operators are defined so as to have the same functional effect in the $a$ th domain, as follows:

$$
\begin{aligned}
\left\{\mathcal{U}_{a} f_{a}\right\}\left(u_{a}\right) & =u_{a} f_{a}\left(u_{a}\right), \\
\left\{\mathcal{D}_{a} f_{a}\right\}\left(u_{a}\right) & =(i 2 \pi)^{-1} f_{a}^{\prime}\left(u_{a}\right) .
\end{aligned}
$$

It follows from these definitions that $\mathcal{U}_{a}$ and $\mathcal{D}_{a}$ satisfy (11) and (12) with the special cases $\mathcal{U}_{0}=\mathcal{U}, \mathcal{U}_{1}=\mathcal{D}, \mathcal{U}_{-1}=-\mathcal{D}$, $\mathcal{D}_{0}=\mathcal{D}, \mathcal{D}_{1}=-\mathcal{U}, \mathcal{D}_{-1}=\mathcal{U}$. The first and fourth of these special cases are by definition. The second and fifth follow from the Fourier transform property mentioned in the previous paragraph and its dual: $\mathcal{D}_{1}=\mathcal{F}^{-1} \mathcal{D} \mathcal{F}=\mathcal{F}^{-1}\left(\mathcal{F}^{-1} \mathcal{U} \mathcal{F}\right) \mathcal{F}=$ $\mathcal{F}^{-2} \mathcal{U F}^{2}=\mathcal{P U P}=-\mathcal{U}$. The third can be obtained by substituting $\mathcal{U}=-\mathcal{D}_{1}=-\mathcal{F}^{-1} \mathcal{D} \mathcal{F}$ into $\mathcal{U}_{-1}=\mathcal{F U F}^{-1}$ (both special cases of (11)), and the sixth can be obtained similarly to the third.

It can be shown that the fractional operators $\mathcal{U}_{a}$ and $\mathcal{D}_{a}$ can be expressed in terms of their integer counterparts as given in entry 1 of Table I. As the fractional order parameter $a$ varies from 0 to 1 , the relative contributions of $\mathcal{U}$ and $\mathcal{D}$ to $\mathcal{U}_{a}$ and $\mathcal{D}_{a}$ are given by simple trigonometric factors.

The phase shift operator $\mathcal{P H}(\eta)$ and the translation operator $\mathcal{S H}(\xi)$ are defined as follows:

$$
\begin{aligned}
\mathcal{P H}(\eta) & =\exp (i 2 \pi \eta \mathcal{U}) \\
\mathcal{S H}(\xi) & =\exp (i 2 \pi \xi \mathcal{D}) .
\end{aligned}
$$

Such expressions are interpreted in terms of their series expansions. These operators shift or translate signals in the frequency and time domains, respectively [1], as follows:

$$
\begin{aligned}
& \{\mathcal{P H}(\eta) f\}(u)=\exp (i 2 \pi \eta u) f(u) \\
& \{\mathcal{S H}(\xi) f\}(u)=f(u+\xi) .
\end{aligned}
$$

These two operators in question are related through (10) either with $\mathcal{A}=\mathcal{P} \mathcal{H}(\xi)$ and $\mathcal{A}^{\mathrm{D}}=\mathcal{S H}(\xi)$, or with $\mathcal{A}=\mathcal{S H}(\xi)$ and $\mathcal{A}^{\mathrm{D}}=\mathcal{P H}(-\xi):[\mathcal{S H}(\xi)]^{D}=\mathcal{F}^{-1} \mathcal{S H}(\xi) \mathcal{F}=$ $\mathcal{F}^{-2} \mathcal{P} \mathcal{H}(\xi) \mathcal{F}^{2}=\mathcal{P} \mathcal{P} \mathcal{H}(\xi) \mathcal{P}=\mathcal{P} \mathcal{H}(-\xi)$

The fractional forms of these operators are defined as

$$
\begin{aligned}
\mathcal{P} \mathcal{H}_{a}(\eta) & =\exp \left(i 2 \pi \eta \mathcal{U}_{a}\right) \\
\mathcal{S H} & (\xi)=\exp \left(i 2 \pi \xi \mathcal{D}_{a}\right) .
\end{aligned}
$$

It can be shown that these operators have the same functional effect in the $a$ th domain as their ordinary counterparts have in the time domain and satisfy (11) and (12). Again, it is possible to express these operators in terms of their integer counterparts, as given in entry 2 of Table I. As the fractional order parameter $a$ varies from 0 to 1 , the relative contributions of $\mathcal{P H}(\eta)$ and $\mathcal{S H}(\xi)$ to $\mathcal{P H}_{a}(\eta)$ and $\mathcal{S H}_{a}(\xi)$ are given by simple trigonometric factors. 
TABLE I

SUMMARY OF FRACTIONAL OPERATORS

\begin{tabular}{|c|c|c|c|}
\hline & Fractional operator & Symbol & Equivalent expression(s) \\
\hline $1-a)$ & Frac. coor. multip. [1], [24], [25] & $\mathcal{U}_{a}$ & $\cos \alpha \mathcal{U}+\sin \alpha \mathcal{D}$ \\
\hline $1-b)$ & Frac. differentiation [1], [24], [25] & $\mathcal{D}_{a}$ & $-\sin \alpha \mathcal{U}+\cos \alpha \mathcal{D}$ \\
\hline $2-a)$ & Frac. phase shift [1], [24], [25] & $\mathcal{P} \mathcal{H}_{a}(\eta)$ & $\begin{array}{l}\exp \left(i \pi \eta^{2} \sin \alpha \cos \alpha\right) \mathcal{P H}(\eta \cos \alpha) \mathcal{S H}(\eta \sin \alpha) \\
\exp \left(-i \pi \eta^{2} \sin \alpha \cos \alpha\right) \mathcal{S H}(\eta \sin \alpha) \mathcal{P} \mathcal{H}(\eta \cos \alpha)\end{array}$ \\
\hline 2-b) & Frac. translation [1], [24], [25] & $\mathcal{S} \mathcal{H}_{a}(\xi)$ & $\begin{array}{l}\exp \left(i \pi \xi^{2} \sin \alpha \cos \alpha\right) \mathcal{S H}(\xi \cos \alpha) \mathcal{P H}(-\xi \sin \alpha) \\
\exp \left(-i \pi \xi^{2} \sin \alpha \cos \alpha\right) \mathcal{P H}(-\xi \sin \alpha) \mathcal{S H}(\xi \cos \alpha)\end{array}$ \\
\hline 3- & Frac. scaling [4] & $\mathcal{M}_{a}(M)$ & $\mathcal{F}^{-a} \mathcal{M}(M) \mathcal{F}^{a}, \mathcal{F}^{-1-a} \mathcal{M}(1 / M) \mathcal{F}^{1+a}$ \\
\hline 4-a) & Frac. chirp multip. [4] & $\mathcal{Q}_{a}(q)$ & $\begin{array}{c}\mathcal{R}(-\tan \alpha) \mathcal{Q}\left(q \cos ^{2} \alpha\right) \mathcal{R}(\tan \alpha) \\
\mathcal{Q}(\cot \alpha) \mathcal{R}\left(q \sin ^{2} \alpha\right) \mathcal{Q}(-\cot \alpha)\end{array}$ \\
\hline 4-b) & Frac. chirp convol. [4] & $\mathcal{R}_{a}(r)$ & $\begin{array}{l}\mathcal{R}(\cot \alpha) \mathcal{Q}\left(r \sin ^{2} \alpha\right) \mathcal{R}(-\cot \alpha) \\
\mathcal{Q}(-\tan \alpha) \mathcal{R}\left(r \cos ^{2} \alpha\right) \mathcal{Q}(\tan \alpha)\end{array}$ \\
\hline $5-a)$ & Frac. discretization & $\mathcal{D} \mathcal{I}_{a}(\Delta \mu)$ & $\begin{array}{c}\mathcal{R}(-\tan \alpha) \mathcal{D} \mathcal{I}(\Delta \mu \cos \alpha) \mathcal{R}(\tan \alpha) \\
\mathcal{Q}(\cot \alpha) \mathcal{P E}(\Delta \mu \sin \alpha) \mathcal{Q}(-\cot \alpha)\end{array}$ \\
\hline $5-b)$ & Frac. periodization & $\mathcal{P} \mathcal{E}_{a}(\Delta u)$ & $\begin{array}{l}\mathcal{R}(\cot \alpha) \mathcal{D I}(\Delta u \sin \alpha) \mathcal{R}(-\cot \alpha) \\
\mathcal{Q}(-\tan \alpha) \mathcal{P} \mathcal{E}(\Delta u \cos \alpha) \mathcal{Q}(\tan \alpha)\end{array}$ \\
\hline
\end{tabular}

The scaling operator $\mathcal{M}(M)$ can be defined through the coordinate multiplication and differentiation operators in the following way:

$$
\mathcal{M}(M)=\exp [-i \pi(\ln M)(\mathcal{U D}+\mathcal{D U})]
$$

where $M>0$. Its effect in the time domain is [29, p. 275]

$$
\{\mathcal{M}(M) f\}(u)=\sqrt{1 / M} f(u / M) .
$$

The scaling operator is its own dual in the sense that scaling in the time domain corresponds to descaling in the frequency domain: the Fourier transform of $\sqrt{1 / M} f(u / M)$ is $\sqrt{M} F(M \mu)$. Once again, this operator satisfies (10) with $\mathcal{A}=\mathcal{M}(M)$ and $\mathcal{A}^{\mathrm{D}}=\mathcal{M}(1 / M)$.

The fractional form of the scaling operator is again defined in the same manner, as follows:

$$
\mathcal{M}_{a}(M)=\exp \left[-i \pi(\ln M)\left(\mathcal{U}_{a} \mathcal{D}_{a}+\mathcal{D}_{a} \mathcal{U}_{a}\right)\right],
$$

This operator has the same functional effect in the ath domain as its ordinary counterpart has in the time domain and satisfies (11) and (12). Once again, it is possible to express this operator in terms of its integer counterparts, as given in entry 3 of Table I. This result expresses the fractional scaling operator in terms of the ordinary scaling operator or its dual (which is also a scaling operator with reciprocal parameter). That (25) satisfies (11) can be shown by first noting that for integer $k$ we have $\left(\mathcal{U}_{a} \mathcal{D}_{a}+\mathcal{D}_{a} \mathcal{U}_{a}\right)^{k}=$ $\left(\mathcal{F}^{-a} \mathcal{U} \mathcal{F}^{a} \mathcal{F}^{-a} \mathcal{D} \mathcal{F}^{a}+\mathcal{F}^{-a} \mathcal{D F}^{a} \mathcal{F}^{-a} \mathcal{U} \mathcal{F}^{a}\right)^{k}=\left[\mathcal{F}^{-a}(\mathcal{U D}+\right.$ $\left.\mathcal{D U}) \mathcal{F}^{a}\right]^{k}=\mathcal{F}^{-a}(\mathcal{U D}+\mathcal{D U})^{k} \mathcal{F}^{a}$. Now, we use this result in the series expansion: $\mathcal{M}_{a}(M)=\sum_{k=0}^{\infty}(-i \pi \ln M)^{k}\left(\mathcal{U}_{a} \mathcal{D}_{a}+\right.$ $\left.\mathcal{D}_{a} \mathcal{U}_{a}\right)^{k} / k !=\mathcal{F}^{-a}\left[\sum_{k=0}^{\infty}(-i \pi \ln M)^{k}(\mathcal{U D}+\mathcal{D U})^{k} / k !\right] \mathcal{F}^{a}=$ $\mathcal{F}^{-a} \mathcal{M}(M) \mathcal{F}^{a}$, which is the desired result. This derivation also applies to (12) since the dual is obtained merely by replacing $M$ by $1 / M$.
The chirp multiplication operator $\mathcal{Q}(q)$ and the chirp convolution operator $\mathcal{R}(r)$ are defined as follows:

$$
\begin{aligned}
& \mathcal{Q}(q)=\exp \left(-i \pi q \mathcal{U}^{2}\right) \\
& \mathcal{R}(r)=\exp \left(-i \pi r \mathcal{D}^{2}\right) .
\end{aligned}
$$

Their effect in the time domain is given by

$$
\begin{aligned}
& \{\mathcal{Q}(q) f\}(u)=\exp \left(-i \pi q u^{2}\right) f(u), \\
& \{\mathcal{R}(r) f\}(u)=\exp (-i \pi / 4) \sqrt{1 / r} \exp \left(i \pi u^{2} / r\right) * f(u)
\end{aligned}
$$

These two operators again form a Fourier dual pair satisfying (10) either with $\mathcal{A}=\mathcal{Q}(q)$ and $\mathcal{A}^{\mathrm{D}}=\mathcal{R}(q)$, or with $\mathcal{A}=$ $\mathcal{R}(r)$ and $\mathcal{A}^{\mathrm{D}}=\mathcal{Q}(r)$, which readily follow from their series expansions:

$$
\begin{aligned}
\mathcal{Q}(q) & =\sum_{k=0}^{\infty} \frac{(-i \pi q)^{k}}{k !} \mathcal{U}^{2 k} \\
& =\sum_{k=0}^{\infty} \frac{(-i \pi q)^{k}}{k !}\left(-\mathcal{F}^{-1} \mathcal{D} \mathcal{F}\right)^{2 k} \\
& =\sum_{k=0}^{\infty} \frac{(-i \pi q)^{k}}{k !} \mathcal{F}^{-1} \mathcal{D}^{2 k} \mathcal{F}=\mathcal{F}^{-1} \mathcal{R}(q) \mathcal{F} \\
\mathcal{Q}(q) & =\sum_{k=0}^{\infty} \frac{(-i \pi q)^{k}}{k !}\left(\mathcal{F} \mathcal{D} \mathcal{F}^{-1}\right)^{2 k} \\
& =\sum_{k=0}^{\infty} \frac{(-i \pi q)^{k}}{k !} \mathcal{F D}^{2 k} \mathcal{F}^{-1}=\mathcal{F} \mathcal{R}(q) \mathcal{F}^{-1} .
\end{aligned}
$$

Multiplication with a chirp may be interpreted as frequency modulation with a linearly varying frequency component. Since $\mathcal{Q}(q)$ and $\mathcal{R}(r)$ are duals satisfying (10), the effect of $\mathcal{R}(r)$ in the time domain must be functionally equivalent to the effect of $\mathcal{Q}(r)$ in the frequency domain. Therefore, $\{\mathcal{R}(r) f\}(u)$ is given 
by the inverse Fourier transform of the frequency domain function $e^{-i \pi r \mu^{2}} F(\mu)$, which can be evaluated using the convolution theorem and the Fourier transform pair $e^{-i \pi / 4} \sqrt{1 / r} e^{i \pi u^{2} / r} \leftrightarrow$ $e^{-i \pi r \mu^{2}}$.

We define the fractional forms of these operators as

$$
\begin{aligned}
\mathcal{Q}_{a}(q) & =\exp \left(-i \pi q \mathcal{U}_{a}^{2}\right) \\
\mathcal{R}_{a}(r) & =\exp \left(-i \pi r \mathcal{D}_{a}^{2}\right) .
\end{aligned}
$$

These operators have the same functional effect in the $a$ th domain as their ordinary counterparts have in the time domain, and satisfy (11) and (12). These fractional chirp operators can be expressed in terms of $\mathcal{Q}(q)$ and $\mathcal{R}(r)$ as given in entry 4 of Table I.

\section{Periodicity AND Discreteness Operators AND Their FRACTIONAL GENERALIZATIONS}

In the previous section, we reviewed common pairs of dual operators and their fractional versions. In this section we turn our attention to the main focus of this paper, which is to explore fractional generalizations of the dual properties of periodicity and discreteness. To facilitate our analysis, we define two operators corresponding to these two properties, such that when one of these operators acts on an arbitrary function, it produces a function with the corresponding property.

We define the discretization $\mathcal{D} \mathcal{I}(\Delta \mu)$ and periodization $\mathcal{P E}(\Delta u)$ operators in terms of the phase shift and translation operators

$$
\begin{aligned}
& \mathcal{D} \mathcal{I}(\Delta \mu)=\sum_{k=-\infty}^{\infty} \mathcal{P H}(k \Delta \mu)=\sum_{k=-\infty}^{\infty} \exp (i 2 \pi k \Delta \mu \mathcal{U}) \\
& \mathcal{P E}(\Delta u)=\sum_{k=-\infty}^{\infty} \mathcal{S H}(k \Delta u)=\sum_{k=-\infty}^{\infty} \exp (i 2 \pi k \Delta u \mathcal{D}) .
\end{aligned}
$$

We will see below that the parameters $\Delta u>0$ and $\Delta \mu>0$ correspond to the period of replication in the time and frequency domains respectively. (Remember that, sampling in the time domain corresponds to periodic replication in the frequency domain.) We also define $\delta u=1 / \Delta \mu$ and $\delta \mu=1 / \Delta u$, denoting the sampling interval in the time and frequency domains, respectively.

From this definition and the duality of the phase shift and translation operators, it immediately follows that these two operators are also Fourier duals:

$$
\begin{aligned}
\mathcal{P E}(\Delta u) & =\sum_{k=-\infty}^{\infty} \mathcal{S H}(k \Delta u) \\
& =\sum_{k=-\infty}^{\infty} \mathcal{F}^{-1} \mathcal{P} \mathcal{H}(k \Delta u) \mathcal{F}=\mathcal{F}^{-1} \mathcal{D} \mathcal{I}(\Delta u) \mathcal{F} \\
\mathcal{P E}(\Delta u) & =\sum_{k=-\infty}^{\infty} \mathcal{S H}(-k \Delta u)=\sum_{k=-\infty}^{\infty} \mathcal{F} \mathcal{P} \mathcal{H}(k \Delta u) \mathcal{F}^{-1} \\
& =\mathcal{F} \mathcal{D} \mathcal{I}(\Delta u) \mathcal{F}^{-1}
\end{aligned}
$$

which follow from (32) and (33), $\sum_{k=-\infty}^{\infty} \mathcal{S H}(-k \Delta u)=$ $\sum_{k=-\infty}^{\infty} \mathcal{S H}(k \Delta u)$, and the duality relations $[\mathcal{P H}(\xi)]^{D}=$ $\mathcal{S H}(\xi),[\mathcal{S H}(\xi)]^{D}=\mathcal{P H}(-\xi)$.
The effects of the discretization and periodization operators in the time domain can be expressed as

$$
\begin{aligned}
\{\mathcal{D I}(\Delta \mu) f\}(u) & =\sum_{k=-\infty}^{\infty} \exp (i 2 \pi k u / \delta u) f(u) \\
& =\delta u \sum_{k=-\infty}^{\infty} \delta(u-k \delta u) f(k \delta u) \\
\{\mathcal{P E}(\Delta u) f\}(u) & =\sum_{k=-\infty}^{\infty} f(u-k \Delta u)
\end{aligned}
$$

where we used another form of Poisson's summation formula [5]

$$
\sum_{n=-\infty}^{\infty} \delta(u-n \delta u)=\frac{1}{\delta u} \sum_{n=-\infty}^{\infty} \exp (i 2 \pi n u / \delta u)
$$

to get (35). The $\operatorname{comb}(u)$ function defined as

$$
\operatorname{comb}(u)=\sum_{k=-\infty}^{\infty} \delta(u-k)=\sum_{k=-\infty}^{\infty} \delta(u+k)
$$

can now be used to rewrite (35) and (36) in the following manner:

$$
\begin{aligned}
& \{\mathcal{D I}(\Delta \mu) f\}(u)=\operatorname{comb}(u \Delta \mu) f(u) \\
& \{\mathcal{P E}(\Delta u) f\}(u)=\frac{1}{\Delta u} \operatorname{comb}\left(\frac{u}{\Delta u}\right) * f(u) .
\end{aligned}
$$

From the above equations, it is apparent that $\mathcal{D I}(\Delta \mu)$ is the operator which multiplies a function in the time domain by a delta train, thereby creating a weighted sequence of impulses. On the other hand, $\mathcal{P} \mathcal{E}(\Delta u)$ is the operator that periodically replicates a function in the time domain.

The Fourier transform of $\Delta u^{-1} \operatorname{comb}(u / \Delta u)$ is $\operatorname{comb}(\Delta u \mu)$. Using this result, the Fourier transforms of the right hand sides of (39) and (40) are, respectively, given by

$$
\begin{aligned}
\frac{1}{\Delta \mu} \operatorname{comb}\left(\frac{\mu}{\Delta \mu}\right) * F(\mu) & =\sum_{k=-\infty}^{\infty} F(\mu-k \Delta \mu) \\
\operatorname{comb}(\Delta u \mu) F(\mu) & =\delta \mu \sum_{k=-\infty}^{\infty} \delta(\mu-k \delta \mu) F(k \delta \mu)
\end{aligned}
$$

from which we observe that the effects of the two operators are exchanged in the frequency domain, with the discretization operator leading to periodic replication and the periodization operator leading to multiplication by a delta train. This is precisely what is meant by the duality of the two operators. The reader might also want to compare (41) and (42) with (35) and (36).

We now define the fractional generalizations of these operators as

$$
\begin{aligned}
\mathcal{D I}_{a}(\Delta \mu) & =\sum_{k=-\infty}^{\infty} \mathcal{P} \mathcal{H}_{a}(k \Delta \mu) \\
& =\sum_{k=-\infty}^{\infty} \exp \left(i 2 \pi k \Delta \mu \mathcal{U}_{a}\right) \\
\mathcal{P E}_{a}(\Delta u) & =\sum_{k=-\infty}^{\infty} \mathcal{S H}_{a}(k \Delta u) \\
& =\sum_{k=-\infty}^{\infty} \exp \left(i 2 \pi k \Delta u \mathcal{D}_{a}\right)
\end{aligned}
$$


where $\mathcal{D} \mathcal{I}_{a}(\Delta \mu)$ is the fractional discretization operator and $\mathcal{P E}_{a}(\Delta u)$ is the fractional periodization operator. We prove in Appendix I that these operators satisfy the form of (11)

$$
\begin{aligned}
& \mathcal{D I}_{a}(\Delta \mu)=\mathcal{F}^{-a} \mathcal{D} \mathcal{I}(\Delta \mu) \mathcal{F}^{a} \\
& \mathcal{P E}_{a}(\Delta u)=\mathcal{F}^{-a} \mathcal{P E}(\Delta u) \mathcal{F}^{a} .
\end{aligned}
$$

Using these equations and (34), we can further show

$$
\begin{aligned}
\mathcal{P E}_{a}(\Delta u) & =\mathcal{F}^{-1} \mathcal{D} \mathcal{I}_{a}(\Delta u) \mathcal{F} \\
& =\mathcal{F D I}_{a}(\Delta u) \mathcal{F}^{-1}
\end{aligned}
$$

The right-hand sides of (43) and (44) can be expanded by using entry 2 of Table I to obtain

$$
\begin{aligned}
\mathcal{D I}_{a}(\Delta \mu)= & \sum_{k=-\infty}^{\infty} \exp \left[i \pi(k \Delta \mu)^{2} \sin \alpha \cos \alpha\right] \\
& \times \mathcal{P H}(k \Delta \mu \cos \alpha) \mathcal{S H}(k \Delta \mu \sin \alpha) \\
= & \sum_{k=-\infty}^{\infty} \exp \left[i \pi(k \Delta \mu)^{2} \sin \alpha \cos \alpha\right] \\
& \times[\mathcal{P H}(\Delta \mu \cos \alpha)]^{k}[\mathcal{S H}(\Delta \mu \sin \alpha)]^{k} \\
\mathcal{P E}_{a}(\Delta u)= & \sum_{k=-\infty}^{\infty} \exp \left[i \pi(k \Delta u)^{2} \sin \alpha \cos \alpha\right] \\
& \times \mathcal{S H}(k \Delta u \cos \alpha) \mathcal{P H}(-k \Delta u \sin \alpha) . \\
= & \sum_{k=-\infty}^{\infty} \exp \left[i \pi(k \Delta u)^{2} \sin \alpha \cos \alpha\right] \\
& {[\mathcal{S H}(\Delta u \cos \alpha)]^{k}[\mathcal{P H}(-\Delta u \sin \alpha)]^{k} }
\end{aligned}
$$

where we used $[\mathcal{P H}(\eta)]^{k}=\mathcal{P H}(k \eta)$ and $[\mathcal{S H}(\xi)]^{k}=$ $\mathcal{S H}(k \xi)$, which can easily be proved from their defining equations.

We now derive an intermediate result for the fractional phase shift operator

$$
\begin{aligned}
\mathcal{P H} & (\eta)= \\
& \exp \left(i \pi \eta^{2} \sin \alpha \cos \alpha\right) \\
= & \operatorname{P} \mathcal{H}(\eta \cos \alpha) \mathcal{S H}(\eta \pi \sin \alpha) \\
& \times \exp (i 2 \pi \eta \cos \alpha \mathcal{U}) \mathcal{S H}(\eta \sin \alpha) \\
= & \exp \left(-i \pi \cot \alpha \mathcal{U}^{2}\right) \\
& \exp \left[i \pi \cot \alpha(\mathcal{U}+\eta \sin \alpha \mathcal{I})^{2}\right] \mathcal{S H}(\eta \sin \alpha) \\
= & \exp \left(-i \pi \cot \alpha \mathcal{U}^{2}\right) \mathcal{S H}(\eta \sin \alpha) \\
& \times \exp \left(i \pi \cot \alpha \mathcal{U}^{2}\right),
\end{aligned}
$$

which means that the fractional phase shift operator can be expressed as

$$
\mathcal{P} \mathcal{H}_{a}(\eta)=\mathcal{Q}(\cot \alpha) \mathcal{S H}(\eta \sin \alpha) \mathcal{Q}(-\cot \alpha) .
$$

Employing a similar technique for the fractional translation operator yields

$$
\mathcal{S H}_{a}(\xi)=\mathcal{Q}(-\tan \alpha) \mathcal{S H}(\xi \cos \alpha) \mathcal{Q}(\tan \alpha) .
$$

Now by substituting (50) in (43), we obtain

$$
\begin{aligned}
\mathcal{D I}_{a}(\Delta \mu) & =\sum_{k=-\infty}^{\infty} \mathcal{Q}(\cot \alpha) \mathcal{S H}(k \Delta \mu \sin \alpha) \mathcal{Q}(-\cot \alpha) \\
& =\mathcal{Q}(\cot \alpha) \sum_{k=-\infty}^{\infty} \mathcal{S H}(k \Delta \mu \sin \alpha) \mathcal{Q}(-\cot \alpha) \\
& =\mathcal{Q}(\cot \alpha) \mathcal{P E}(\Delta \mu \sin \alpha) \mathcal{Q}(-\cot \alpha)
\end{aligned}
$$

Likewise, substituting (51) in (44) yields

$$
\mathcal{P} \mathcal{E}_{a}(\Delta u)=\mathcal{Q}(-\tan \alpha) \mathcal{P E}(\Delta u \cos \alpha) \mathcal{Q}(\tan \alpha) .
$$

We can obtain two more equations by using the duality of $\mathcal{Q}$ and $\mathcal{R}$, and $\mathcal{P H}$ and $\mathcal{S H}$

$$
\begin{aligned}
\mathcal{D I}_{a}(\Delta \mu) & =\mathcal{Q}(\cot \alpha) \mathcal{F}^{-1} \mathcal{D} \mathcal{I}(\Delta \mu \sin \alpha) \mathcal{F} \mathcal{Q}(-\cot \alpha) \\
\mathcal{D} \mathcal{I}_{a}(\Delta \mu) & =\mathcal{F}^{-1} \mathcal{R}(\cot \alpha) \mathcal{D} \mathcal{I}(\Delta \mu \sin \alpha) \mathcal{R}(-\cot \alpha) \mathcal{F} \\
\mathcal{F D I}_{a}(\Delta \mu) \mathcal{F}^{-1} & =\mathcal{R}(\cot \alpha) \mathcal{D I}(\Delta \mu \sin \alpha) \mathcal{R}(-\cot \alpha) \\
\mathcal{P} \mathcal{E}_{a}(\Delta u) & =\mathcal{R}(\cot \alpha) \mathcal{D I}(\Delta u \sin \alpha) \mathcal{R}(-\cot \alpha)
\end{aligned}
$$

where we used (34) and $\left(\mathcal{A}^{\mathrm{D}}\right)_{a}=\left(\mathcal{A}_{a}\right)^{\mathrm{D}}$. Similarly

$$
\begin{aligned}
\mathcal{P} \mathcal{E}_{a}(\Delta u) & =\mathcal{Q}(-\tan \alpha) \mathcal{F}^{-1} \mathcal{D} \mathcal{I}(\Delta u \cos \alpha) \mathcal{F} \mathcal{Q}(\tan \alpha) \\
\mathcal{P E} \mathcal{E}_{a}(\Delta u) & =\mathcal{F}^{-1} \mathcal{R}(-\tan \alpha) \mathcal{D} \mathcal{I}(\Delta u \cos \alpha) \mathcal{R}(\tan \alpha) \mathcal{F} \\
\mathcal{F P E}_{a}(\Delta u) \mathcal{F}^{-1} & =\mathcal{R}(-\tan \alpha) \mathcal{D I}(\Delta u \cos \alpha) \mathcal{R}(\tan \alpha) \\
\mathcal{D} \mathcal{I}_{a}(\Delta \mu) & =\mathcal{R}(-\tan \alpha) \mathcal{D I}(\Delta \mu \cos \alpha) \mathcal{R}(\tan \alpha) .
\end{aligned}
$$

Equation (52)-(55) express the fractional discretization and periodization operators in terms of their nonfractional counterparts, revealing that the fractional periodization/discretization operators can be implemented by using only ordinary chirp and ordinary periodization or discretization operations. The fractional operators interpolate between periodicity and discreteness with the smooth transition being governed by the parameter $a$. Therefore, these equations have been included alongside other similar formulas in Table I.

It is worth noting the similarity between entry 4 of Table I and (52)-(55). These eight equations are repeated for easy comparison:

$$
\begin{aligned}
\mathcal{Q}_{a}(q) & =\mathcal{R}(-\tan \alpha) \mathcal{Q}\left(q \cos ^{2} \alpha\right) \mathcal{R}(\tan \alpha) \\
\mathcal{D I}_{a}(\Delta \mu) & =\mathcal{R}(-\tan \alpha) \mathcal{D} \mathcal{I}(\Delta \mu \cos \alpha) \mathcal{R}(\tan \alpha) \\
\mathcal{R}_{a}(r) & =\mathcal{R}(\cot \alpha) \mathcal{Q}\left(r \sin ^{2} \alpha\right) \mathcal{R}(-\cot \alpha) \\
\mathcal{P} \mathcal{E}_{a}(\Delta u) & =\mathcal{R}(\cot \alpha) \mathcal{D} \mathcal{I}(\Delta u \sin \alpha) \mathcal{R}(-\cot \alpha) \\
\mathcal{Q}_{a}(q) & =\mathcal{Q}(\cot \alpha) \mathcal{R}\left(q \sin ^{2} \alpha\right) \mathcal{Q}(-\cot \alpha) \\
\mathcal{D I}_{a}(\Delta \mu) & =\mathcal{Q}(\cot \alpha) \mathcal{P E}(\Delta \mu \sin \alpha) \mathcal{Q}(-\cot \alpha) \\
\mathcal{R}_{a}(r) & =\mathcal{Q}(-\tan \alpha) \mathcal{R}\left(r \cos ^{2} \alpha\right) \mathcal{Q}(\tan \alpha) \\
\mathcal{P} \mathcal{E}_{a}(\Delta u) & =\mathcal{Q}(-\tan \alpha) \mathcal{P E}(\Delta u \cos \alpha) \mathcal{Q}(\tan \alpha) .
\end{aligned}
$$

Notice especially the similar appearance of the chirp multiplication and convolution operators on the right-hand sides of the equations. Although one may be tempted to think that similar relations are satisfied between all dual pairs and their fractional versions, in Appendix II, we show that this is not the case by considering the fractional scaling operator $\mathcal{M}_{a}(M)$. 
We also note that the discretization and periodization operators do not in general have left-hand inverses. The effects of the discretization and periodization operators may be undone only if the function under consideration is of finite extent in the frequency domain (for discretization) or time domain (for periodization). The first of these corresponds to the standard sampling theorem. In other cases, it is impossible to reconstruct the original signal. These operators also do not, in general, have right-hand inverses. If $f(u)$ is not discrete or periodic to begin with, with whatever operation we precede the discretization or periodization operator, we cannot recover $f(u)$. Notice that these properties of the discretization and periodization operators contrast with all the other dual operators and their fractional versions discussed earlier, all of which have inverses.

For completeness, we also present the explicit functional formulas for the effects of fractional periodization and discretization operators acting on a function $f(u)$

$$
\begin{aligned}
\mathcal{P E}_{a}(\Delta u)[f(u)] & \\
= & \sum_{k=-\infty}^{\infty} \exp \left(-i \pi k^{2} \Delta u^{2} \sin \alpha \cos \alpha\right) \\
\quad & \times \exp (i 2 \pi k \Delta u \sin \alpha u) f(u-k \Delta u \cos \alpha) \\
\mathcal{D} \mathcal{I}_{a}(\Delta \mu)[f(u)] & \\
= & \sum_{k=-\infty}^{\infty} \exp \left(i \pi k^{2} \Delta \mu^{2} \sin \alpha \cos \alpha\right) \\
\quad & \times \exp (-i 2 \pi k \Delta \mu \cos \alpha u) f(u-k \Delta \mu \sin \alpha) .
\end{aligned}
$$

The discretization and periodization operators and their fractional versions are somewhat different than the other operators discussed in that these are not operators which we usually simulate with discrete versions; rather, it is these operations which constitute the bridge between continuous and finite signals.

\section{Partial Periodization AND Discretization OPERATORS}

The expressions in Table I show how the fractional operators defined can be expressed in terms of their non-fractional ordinary counterparts. Entry 1 of the table shows us how $\mathcal{U}_{a}$ and $\mathcal{D}_{a}$ can be written as linear combinations of $\mathcal{U}$ and $\mathcal{D}$. The fractional operator $\mathcal{U}_{a}$ consists partially of $\mathcal{U}$ and partially of $\mathcal{D}$, the amount of the contributions being given by trigonometric factors. Entry 2 shows us how $\mathcal{P} \mathcal{H}_{a}$ and $\mathcal{S H}_{a}$ can be written in terms of $\mathcal{P H}$ and $\mathcal{S H}$. The fractional operator $\mathcal{P H} \mathcal{H}_{a}$ consists partially of $\mathcal{P H}$ and $\mathcal{S H}$. This time, however, rather than a linear combination, the amount of the contributions are determined by the parameters of these operators. Entry 4 shows a similar relation for the chirp multiplication and convolution operators, with similar interpretation.

From these observations, we would expect the effect of $\mathcal{D} \mathcal{I}_{a}$ to consist of partial contributions from $\mathcal{D I}$ and $\mathcal{P E}$. What is not initially clear is what it means for a function to be partially discrete or periodic. In the case of $\mathcal{U}$ and $\mathcal{D}$, partialness was obtained by multiplying with scalars of magnitude less than unity. That this will not do for the case of discretization and periodization is obvious. In the case of $\mathcal{P H}$ and $\mathcal{S H}$, partialness was obtained by multiplying the translation or phase shift parameter with scalars of magnitude less than unity. This may lead us to inquire whether partial discretization or partial periodization can be obtained by adjusting the period or sampling interval to vary between its original value and zero or infinity. However, we have found this not to be a satisfactory way of defining the concept of partial discretization or partial periodization.

To make some of the above observations more precise, we state two propositions, whose proofs are given in Appendix III. The first proposition shows that it is not possible to write the fractional discretization or periodization operators as linear combinations of the nonfractional discretization and periodization operators:

The fractional operator $\mathcal{D I}_{a}(\Delta \mu)$ or $\mathcal{P} \mathcal{E}_{a}(\Delta u)$ cannot be written as some finite weighted sum of $\mathcal{D} \mathcal{I}\left(\Delta \mu_{i}\right)$, $\mathcal{P E}\left(\Delta u_{j}\right)$ for any $\Delta \mu_{i}, \Delta u_{j}>0$, where $i, j=1,2, \ldots$

The second proposition shows that it is not possible to write the fractional discretization or periodization operators as product forms of the non-fractional discretization and periodization operators:

The fractional operator $\mathcal{D I}_{a}(\Delta \mu)$ or $\mathcal{P} \mathcal{E}_{a}(\Delta u)$ cannot be written as some finite product of $\mathcal{D} \mathcal{I}\left(\Delta \mu_{i}\right), \mathcal{P} \mathcal{E}\left(\Delta u_{j}\right)$ and a scalar for any $\Delta \mu_{i}, \Delta u_{j}>0$, where $i, j=1,2, \ldots$.

These two propositions suggest that other means of defining partialness should be considered.

To motivate our definition of the partial periodization operator, consider the following expression, which is a truncated version of the "complete" periodization operator defined in (33):

$$
\sum_{k=-(M-1) / 2}^{(M-1) / 2} \mathcal{S H}(k \Delta u) .
$$

When the odd integer $M \rightarrow \infty$, this operator approaches the complete periodization operator. When $M=1$, this operator reduces to the identity operator which does not result in any periodic replication of the function to which it is applied. For a signal $f(u)$ whose energy is mostly concentrated in an interval of extent $\Delta u$ centered around the origin, the effect of the above defined operator may be approximately expressed as

$$
\approx \operatorname{rect}\left(\frac{u}{M \Delta u}\right) \cdot(\mathcal{P E}(\Delta u)[f(u)])
$$

where $\operatorname{rect}(u)=1$ when $|u|<1 / 2$ and 0 elsewhere.

Again, to motivate our definition of the partial discretization operator, let us consider the dual of the operator defined in (58)

$$
\mathcal{F}\left[\sum_{k=-(M-1) / 2}^{(M-1) / 2} \mathcal{S} \mathcal{H}(k \Delta \mu)\right] \mathcal{F}^{-1}=\sum_{k=-(M-1) / 2}^{(M-1) / 2} \mathcal{P} \mathcal{H}(k \Delta \mu)
$$

which is a truncated version of the "complete" discretization operator defined in (32). When $M \rightarrow \infty$, this operator approaches the complete discretization operator. When $M=1$, this operator reduces to the identity operator which does not result in any discretization of the function to which it is applied. For a signal 
$f(u)$ whose inverse Fourier transform energy is mostly concentrated in an interval of extent $\Delta \mu$ centered around the origin, the effect of the above defined operator may be approximately expressed as

$$
\approx M \Delta \mu \operatorname{sinc}(M \Delta \mu u) *(\mathcal{D I}(\Delta \mu)[f(u)])
$$

which is the dual of (59) (multiplication with a rectangle function corresponds to convolution with a sinc function, where $(\operatorname{sinc}(u)=\sin (\pi u) /(\pi u))$. We see that the effect of the operator defined in (60) is to broaden the sampling impulses, corresponding to a loss of resolution.

While the operators defined in (58) and (60) could constitute a self-consistent pair of definitions for the partial periodization and discretization operators respectively, the use of a rectangle-sinc function pair is undesirable in certain respects. One reason is the sharp transition of the rectangle function. Furthermore, it would be desirable to choose a function whose Fourier transform is of the same form as itself to obtain a symmetric formulation. While there are many such functions, our choice here will be the chirp function because of the important role it plays in the theory of the fractional Fourier transform: The fractional Fourier transform of a chirp function is, in general, another chirp function, with the delta function and the harmonic function being limiting special cases [1, p. 149]. While the magnitude of the chirp function is unity for all $u$, it effectively behaves as a convolutive or multiplicative window function since i) the chirp function tends to a delta function as the chirp parameter $1 / r$ goes to infinity, just like a Gaussian function

$$
\lim _{r \rightarrow 0} e^{-i \pi / 4} \sqrt{\frac{1}{r}} \exp \left(i \pi u^{2} / r\right)=\delta(u),
$$

ii) for finite values of the chirp parameter, the tails of the chirp function oscillate so fast that it effectively behaves like a function which is non-zero only over a finite interval, and iii) the chirp function tends to the constant value of 1 as the chirp parameter $q$ goes to zero, again like a Gaussian

$$
\lim _{q \rightarrow 0} \exp \left(-i \pi q u^{2}\right)=1 .
$$

Therefore, we define the partial periodization operator $\mathcal{P E}(\Delta u, s)$ in terms of the chirp multiplication operator as

$$
\mathcal{P E}(\Delta u, s)=\mathcal{Q}\left(\frac{1}{s \Delta u^{2}}\right) \mathcal{P E}(\Delta u)
$$

whose effect on a function $f(u)$ is

$$
\mathcal{P} \mathcal{E}(\Delta u, s)[f(u)]=\exp \left(-i \pi \frac{u^{2}}{s \Delta u^{2}}\right) \cdot(\mathcal{P E}(\Delta u)[f(u)])
$$

which is similar to (59) apart from the fact that a chirp "window" has replaced the rectangular window. Here, $\sqrt{|s|}>0$ is the parameter characterizing the degree of partialness. It tends to infinity when we have complete periodization with an infinite number of periods, and equals 1 when only one period is retained. In general, $\sqrt{|s|}$ can be roughly interpreted as the number of periods retained.
The partial discretization operator $\mathcal{D} \mathcal{I}(\Delta \mu, t)$ is defined as the dual of the partial periodization operator and involves the chirp convolution operator

$$
\begin{aligned}
\mathcal{D I}(\Delta \mu, t) & =\mathcal{F} \mathcal{P} \mathcal{E}(\Delta \mu, t) \mathcal{F}^{-1} \\
& =\mathcal{F} \mathcal{Q}\left(\frac{1}{t \Delta \mu^{2}}\right) \mathcal{P E}(\Delta \mu) \mathcal{F}^{-1} \\
& =\mathcal{R}\left(\frac{1}{t \Delta \mu^{2}}\right) \mathcal{F} \mathcal{P} \mathcal{E}(\Delta \mu) \mathcal{F}^{-1} \\
& =\mathcal{R}\left(\frac{1}{t \Delta \mu^{2}}\right) \mathcal{D} \mathcal{I}(\Delta \mu)
\end{aligned}
$$

whose effect on a function $f(u)$ is

$$
\begin{aligned}
& \mathcal{D} \mathcal{I}(\Delta \mu, t)[f(u)] \\
& \quad=e^{-i \pi / 4} \sqrt{t} \Delta \mu \exp \left[i \pi t(\Delta \mu u)^{2}\right] *(\mathcal{D I}(\Delta \mu)[f(u)]) .
\end{aligned}
$$

Here, $\sqrt{|t|}>0$ is the parameter characterizing the degree of discretization. It tends to infinity when we have complete discretization with ideal delta samples, and equals 1 when the width of the sampling pulses approximately equals the sampling interval. In general, $\sqrt{|t|}$ can be roughly interpreted as the ratio of the sampling interval to the width of the sampling pulse.

Thus, we have defined partial periodization as complete periodization followed by chirp multiplication (which effectively windows the number of periodic replicas) and partial discretization as complete discretization followed by chirp convolution (which effectively broadens the sampling impulses). The parameters of the chirp multiplication and convolution operations determine the degree of partialness. By using (52)-(55), we can write the following relations between partial periodization and discretization operators and the fractional periodization and discretization operators defined earlier:

$$
\begin{aligned}
& \mathcal{D} \mathcal{I}_{a}(\Delta \mu)=\mathcal{D} \mathcal{I}\left(\Delta \mu \cos \alpha,-2 \delta u^{2} / \sin 2 \alpha\right) \mathcal{R}(\tan \alpha) \\
& \mathcal{P} \mathcal{E}_{a}(\Delta u)=\mathcal{D} \mathcal{I}\left(\Delta u \sin \alpha, 2 \delta \mu^{2} / \sin 2 \alpha\right) \mathcal{R}(-\cot \alpha) \\
& \mathcal{D} \mathcal{I}_{a}(\Delta \mu)=\mathcal{P} \mathcal{E}\left(\Delta \mu \sin \alpha, 2 \delta u^{2} / \sin 2 \alpha\right) \mathcal{Q}(-\cot \alpha) \\
& \mathcal{P} \mathcal{E}_{a}(\Delta u)=\mathcal{P} \mathcal{E}\left(\Delta u \cos \alpha,-2 \delta \mu^{2} / \sin 2 \alpha\right) \mathcal{Q}(\tan \alpha) .
\end{aligned}
$$

These relations show how the fractional periodization and discretization operators can be expressed in terms of nonfractional partial periodization and discretization operators. As such, these equations, together with (52)-(55), are of similar significance as those in Table I. They express fractional operators in terms of their nonfractional counterparts.

Observing the general form of the above four equations tempts us to eliminate the operators $\mathcal{Q}$ or $\mathcal{R}$ from the right-hand side by absorbing them in the definition of the partial periodization and discretization operators. A partial periodization operator thus defined would involve multiplication with a window not only after ordinary periodization, but before as well. Likewise, a partial discretization operator thus defined would involve convolution with a window not only after ordinary discretization, but before as well. If the function to be periodized was negligible outside an interval of length $\sqrt{|s|} \Delta u$ around the origin, or the function to be discretized had a negligible Fourier content outside an interval of length 
$\sqrt{|t|} \Delta \mu$ around the origin, these definitions would be nearly identical to those given in (64) and (66). If the function or its Fourier transform are not negligible outside an interval of length $\sqrt{|s|} \Delta u$ or $\sqrt{|t|} \Delta \mu$, respectively, these definitions will differ from those given in (64) and (66), essentially because they clip the tails of the function (beyond $\sqrt{|s|} \Delta u$ ) or its Fourier transform (beyond $\sqrt{|t|} \Delta \mu$ ) prior to periodic replication or sampling. These definitions also satisfy duality and coincide with complete periodization or complete discretization as $\sqrt{|s|}$ or $\sqrt{|t|}$ respectively go to infinity. If these definitions are employed, we obtain the following set of particularly simple relations between fractional periodization and discretization and partial non-fractional periodization and discretization:

$$
\begin{aligned}
& \mathcal{D} \mathcal{I}_{a}(\Delta \mu)=\mathcal{D I}\left(\Delta \mu \cos \alpha,-2 \delta u^{2} / \sin 2 \alpha\right) \\
& \mathcal{P} \mathcal{E}_{a}(\Delta u)=\mathcal{D} \mathcal{I}\left(\Delta u \sin \alpha, 2 \delta \mu^{2} / \sin 2 \alpha\right) \\
& \mathcal{D} \mathcal{I}_{a}(\Delta \mu)=\mathcal{P} \mathcal{E}\left(\Delta \mu \sin \alpha, 2 \delta u^{2} / \sin 2 \alpha\right) \\
& \mathcal{P} \mathcal{E}_{a}(\Delta u)=\mathcal{P} \mathcal{E}\left(\Delta u \cos \alpha,-2 \delta \mu^{2} / \sin 2 \alpha\right)
\end{aligned}
$$

which simply say that fractional periodization or discretization can be interpreted as partial nonfractional periodization or discretization with another period or sampling interval. These relations provide an alternative means of interpreting fractional periodization and discretization operators, compared with (52)-(55).

We can also define fractional versions of the partial periodization and discretization operators

$$
\begin{aligned}
& \mathcal{P} \mathcal{E}_{a}(\Delta u, s)=\mathcal{Q}_{a}\left(\frac{1}{s \Delta u^{2}}\right) \mathcal{P} \mathcal{E}_{a}(\Delta u) \\
& \mathcal{D I}_{a}(\Delta \mu, t)=\mathcal{R}_{a}\left(\frac{1}{t \Delta \mu^{2}}\right) \mathcal{D I}_{a}(\Delta \mu) .
\end{aligned}
$$

These operators, which are both fractional and partial, are also Fourier duals

$$
\mathcal{P E} \mathcal{E}_{a}(\Delta u, s)=\mathcal{F}^{-1} \mathcal{D} \mathcal{I}_{a}(\Delta u, s) \mathcal{F} .
$$

\section{DISCUSSION AND CONCLUSION}

A variety of Fourier dual (or conjugate) operators play important roles both in the theory of the Fourier transform and the time-frequency plane, and in certain areas including quantum mechanics, optics, and signal analysis and processing. Common examples of these operators are coordinate multiplication and differentiation, translation and phase shift, chirp multiplication and convolution, and scaling (which is self-dual). The fractional Fourier transform allows us to define a continuum of operators which interpolate between such dual pairs. These gradually evolve from one member of the dual pair to the other as the fractional order goes from zero to one. Several of these operators and their fractional versions were discussed in previous literature (see [4] and the references therein).

The concepts of periodicity and discreteness are also Fourier duals in the same sense. The Fourier transform of a periodic function is discrete and the Fourier transform of a discrete function is periodic. (Here, the term discrete refers to an impulse train sampled function.) In this paper, we have discussed the interpolation between the dual properties of periodicity and discreteness, showing how one evolves into the other as the order goes from zero to one. In order to base our analysis in the same framework as that used to discuss the dual operators mentioned above, we defined the periodization and discretization operators, whose effect is to create periodic and discrete functions respectively, when applied to an arbitrary function. The definition of the periodization and discretization operators are based on the translation and phase shift operators, the latter which constitute a dual pair themselves. We defined fractional periodization and discretization operators in the same manner as other fractional dual operators have been defined - they all have the same effect in the $a$ th fractional Fourier domain as their ordinary counterparts have in the zeroth (time) domain.

A common goal in dealing with fractional operators is to show how they are related to their non-fractional counterparts (Table I). We have derived various expressions relating the fractional periodization and discretization operators to their nonfractional counterparts. With some dual operators, such as in entry 1 of the table, the fractional operators are given as linear superpositions of the non-fractional operators. With others, as in entry 2 , the parameters of the non-fractional operators take values reflecting their partial effect. For instance, fractional translation will involve partial translation and partial phase shifting. In the case of fractional periodization and discretization, it seems that neither of these is the proper way for a full periodization or discretization operator to make a partial contribution to a fractional periodization or discretization operator. We argue that a suitable way of partializing the periodization operator is to window a finite number of periods and a suitable way of partializing the discretization operator is to smooth an impulse sampled function. With these definitions, the fractional periodization and discretization operators can be related to partial nonfractional periodization and discretization operators in a relatively simple manner. As explained in detail in Section V, we chose to work with chirp windows which in our opinion lead to the analytically most elegant and symmetric formulation. However, in certain situations, especially involving applications, one may be willing to sacrifice symmetry in favor of other objectives, and standard window functions such as Hamming, Kaiser, Hanning, etc., may be preferred. Perhaps the most important observation regarding the concepts of partial periodicity and discreteness is that they model real-life sampled and periodic functions. In real life ideal delta sampled functions and infinite periodic functions do not exist. The sampling pulse is finite, reflecting a finite resolution, and only a finite number of periods can exist. Since we have shown that fractional periodization and discretization operators are related to partial operators, the fractional periodization and discretization operators are also related to real-life sampled and periodic functions.

The definitions and relations given in this paper may be useful especially when dealing with signals with irregular time-frequency support. If a set of signals has a time-frequency support which is a rectangle orthogonal to the coordinate axes, the time-bandwidth product, the area of the rectangular support, and the number of degrees of freedom are roughly equal. However, if the support is not orthogonal to the axes (especially if it has a large aspect ratio), or if it is completely irregular, the time-bandwith product and the number of degrees of freedom may significantly differ. This is particularly noticeable for certain signals, 
such as those of a chirped nature. For instance, when sampling such a signal, sampling at the conventional Nyquist rate over some finite interval will produce a number of samples equal to the time-bandwidth product, whereas if we were to sample the signal in a fractional domain where the rectangle bounding the time-frequency support is smallest, the signal can be represented with fewer samples. Fractional periodicity may be useful in multiplexing signals. Multiplexing in either the time or frequency domain often involves adjacently situating signals which exhibit similar characteristics. While this does not create strictly periodically replicated signals, it is possible to associate the time slot or frequency band used in multiplexing with the replication period in our formulation. If the set of signals has a support like that described above, straightforward multiplexing will be inefficient since the support of each signal will occupy only a certain part of the time-frequency "tile" allocated to it. Again working in the fractional domain where the bounding rectangle is smallest (or possibly even considering hexagonal etc. packing strategies), more efficient time or frequency multiplexing making better use of the channel is possible. Other applications may be based on the relationship of fractional or partial periodicity or discreteness operators to real-world sampled or periodic functions which involve finite-size pulses or a finite number of periods.

\section{APPENDIX I}

The fractional discretization and periodization operators satisfy the form of (11)

$$
\begin{aligned}
& \mathcal{D} \mathcal{I}_{a}(\Delta \mu)=\mathcal{F}^{-a} \mathcal{D} \mathcal{I}(\Delta \mu) \mathcal{F}^{a} \\
& \mathcal{P E} \mathcal{E}_{a}(\Delta u)=\mathcal{F}^{-a} \mathcal{P E}(\Delta u) \mathcal{F}^{a} .
\end{aligned}
$$
we find

Proof: Using (32), (43), and $\mathcal{P} \mathcal{H}_{a}(\eta)=\mathcal{F}^{-a} \mathcal{P} \mathcal{H}(\eta) \mathcal{F}^{a}$,

$$
\begin{aligned}
\mathcal{D I}_{a}(\Delta \mu) & =\sum_{k=-\infty}^{\infty} \mathcal{P} \mathcal{H}_{a}(k \Delta \mu) \\
& =\sum_{k=-\infty}^{\infty} \mathcal{F}^{-a} \mathcal{P} \mathcal{H}(k \Delta \mu) \mathcal{F}^{a} \\
& =\mathcal{F}^{-a}\left[\sum_{k=-\infty}^{\infty} \mathcal{P H}(k \Delta \mu)\right] \mathcal{F}^{a}=\mathcal{F}^{-a} \mathcal{D} \mathcal{I}(\Delta \mu) \mathcal{F}^{a}
\end{aligned}
$$

which is the desired result. The expression for the fractional periodization operator is proved in exactly the same way.

\section{APPENDIX II}

The fractional scaling operator $\mathcal{M}_{a}(M)$ with given $a$ and $M$ cannot be expressed in either of the following forms for any $\phi, M^{\prime}, \chi$ :

$$
\begin{aligned}
& \mathcal{M}_{a}(M)=\mathcal{R}(\phi) \mathcal{M}\left(M^{\prime}\right) \mathcal{R}(\chi) \\
& \mathcal{M}_{a}(M)=\mathcal{Q}(\phi) \mathcal{M}\left(M^{\prime}\right) \mathcal{Q}(\chi) .
\end{aligned}
$$

Proof: The proof follows by using the matrix representations of the operators. When the matrix representations of linear canonical transformation theory are substituted in the above equations [4], it can be shown that for the above forms to be satisfied, we must have $(M-1 / M) \sin \alpha \cos \alpha=0$, which shows that these forms cannot be satisfied for arbitrary $M$ and $a$. (For a general reference on linear canonical transforms, see [29].)

\section{APPENDIX III}

Here, we prove the two quoted propositions in Section V:

The fractional operator $\mathcal{D I}_{a}(\Delta \mu)$ or $\mathcal{P} \mathcal{E}_{a}(\Delta u)$ cannot be written as some finite weighted sum of $\mathcal{D} \mathcal{I}\left(\Delta \mu_{i}\right)$, $\mathcal{P E}\left(\Delta u_{j}\right)$ for any $\Delta \mu_{i}, \Delta u_{j}>0$, where $i, j=1,2, \ldots$.

Proof: Assume that such a form exists for $\mathcal{D I}_{a}(\Delta \mu)$, as follows:

$$
\mathcal{D I}_{a}(\Delta \mu)=\sum_{i=1}^{n} c_{i} \mathcal{D} \mathcal{I}\left(\Delta \mu_{i}\right)+\sum_{j=1}^{m} c_{j}^{\prime} \mathcal{P} \mathcal{E}\left(\Delta u_{j}\right)
$$

for some $n, m \in \mathbb{Z}^{+}$, and some scalars $c_{i}, c_{j}^{\prime}$. Then,

$$
\begin{aligned}
& \left\{\mathcal{D I}_{a}(\Delta \mu) f\right\}(u) \\
& =\left\{\sum_{i=1}^{n} c_{i} \mathcal{D} \mathcal{I}\left(\Delta \mu_{i}\right)+\sum_{j=1}^{m} c_{j}^{\prime} \mathcal{P} \mathcal{E}\left(\Delta u_{j}\right)\right\}[f(u)] .
\end{aligned}
$$

The right-hand side has impulsive components in the time and/or frequency domains while the left-hand side does not, for some $f$, as can be deduced from (52)-(55). This contradiction completes the proof for $\mathcal{D} \mathcal{I}_{a}$. The same result is valid for $\mathcal{P} \mathcal{E}_{a}$, which can be shown in exactly the same way.

The fractional operator $\mathcal{D I}_{a}(\Delta \mu)$ or $\mathcal{P} \mathcal{E}_{a}(\Delta u)$ cannot be written as some finite product of $\mathcal{D} \mathcal{I}\left(\Delta \mu_{i}\right), \mathcal{P E}\left(\Delta u_{j}\right)$ and a scalar for any $\Delta \mu_{i}, \Delta u_{j}>0$, where $i, j=1,2, \ldots$.

Proof: Assume that such a form exists for $\mathcal{D I}_{a}(\Delta \mu)$, as follows:

$$
\begin{aligned}
\mathcal{D} \mathcal{I}_{a}(\Delta \mu)=c \cdot \Gamma\left(\mathcal{D I}\left(\Delta \mu_{1}\right), \ldots,\right. & \\
& \left.\mathcal{D} \mathcal{I}\left(\Delta \mu_{n}\right), \mathcal{P E}\left(\Delta u_{1}\right), \ldots, \mathcal{P} \mathcal{E}\left(\Delta u_{m}\right)\right)
\end{aligned}
$$

for some $n, m \in \mathbb{Z}^{+}$, some scalar $c$, and some product function $\Gamma$ multiplying its arguments in one of their possible permutations. Then

$$
\begin{aligned}
\left\{\mathcal{D I}_{a}(\Delta \mu) f\right\}(u)=c \cdot \Gamma\left(\mathcal{D I}\left(\Delta \mu_{1}\right), \ldots, \mathcal{D I}\left(\Delta \mu_{n}\right),\right. & \\
& \left.\mathcal{P E}\left(\Delta u_{1}\right), \ldots, \mathcal{P E}\left(\Delta u_{m}\right)\right)[f(u)] .
\end{aligned}
$$

The right-hand side has impulsive components in the time and/or frequency domains while the left-hand side does not, for some $f$. This contradiction completes the proof for $\mathcal{D} \mathcal{I}_{a}$. The same result is valid for $\mathcal{P} \mathcal{E}_{a}$, which can be shown in exactly the same way.

Extending the proof to the case where the product terms are raised to arbitrary real powers would be desirable, but is currently not available. 


\section{ACKNOWLEDGMENT}

The authors would like to thank L. Barker of Bilkent University for his suggestions. U. Sümbül would like to thank J. Pauly of Stanford University for his support.

\section{REFERENCES}

[1] H. M. Ozaktas, Z. Zalevsky, and M. A. Kutay, The Fractional Fourier Transform with Applications in Optics and Signal Processing. New York: Wiley, 2001

[2] A. Lohmann, "Duality in optics," Optik, vol. 89, pp. 93-97, 1992.

[3] A. Papoulis, Systems and Transformations with Applications in Optics.. New York: McGraw-Hill, 1968.

[4] U. Sümbül and H. M. Ozaktas, "Fractional free space, fractional lenses, and fractional imaging systems," J. Opt. Soc. Amer. A, vol. 20, no. 11, pp. 2033-2040, 2003.

[5] A. Papoulis, Signal Analysis. New York: McGraw-Hill, 1977.

[6] R. J. Marks II, Introduction to Shannon Sampling and Interpolation Theory.. New York: Springer, 1991

[7] R. J. Marks II, Ed., Advanced Topics in Shannon Sampling and Interpolation Theory. New York: Springer, 1993.

[8] X.-G. Xia, "On bandlimited signals with fractional Fourier transform," IEEE Signal Process. Lett., vol. 3, no. 3, pp. 72-74, 1996.

[9] A. I. Zayed and A. G. Garcia, "New sampling formulae for the fractional Fourier transform," Signal Process., vol. 77, no. 1, pp. 111-114, 1999.

[10] T. Erseghe, P. Kraniauskas, and G. Cariolaro, "Unified fractional Fourier transform and sampling theorem," IEEE Trans. Signal Process., vol. 47, no. 12, pp. 3419-3423, Dec. 1999.

[11] L. Onural, "Sampling of the diffraction field," Appl. Opt., vol. 39, no. 32, pp. 5929-5935, 2000

[12] T. Alieva and A. M. Barbé, "Fractional Fourier and Radon-Wigner transforms of periodic signals," Signal Process., vol. 69, no. 2, pp. 183-189, 1998.

[13] S. C. Pei, M. H. Yeh, and T. L. Luo, "Fractional Fourier series expansion for finite signals and dual extension to discrete-time fractional Fourier transform," IEEE Trans. Signal Process., vol. 47, no. 10, pp. 2883-2888, Oct. 1999.

[14] D. Mendlovic and H. M. Ozaktas, "Fractional Fourier transforms and their optical implementation: I," J. Opt. Soc. Amer. A, vol. 10, no. 9, pp. 1875-1881, 1993.

[15] H. M. Ozaktas and D. Mendlovic, "Fractional Fourier transforms and their optical implementation: II," J. Opt. Soc. Amer. A, vol. 10, no. 12, pp. 2522-2531, 1993.

[16] A. W. Lohmann, "Image rotation, Wigner rotation, and the fractional order Fourier transform," J. Opt. Soc. Amer. A, vol. 10, no. 10, pp. 2181-2186, 1993

[17] H. M. Ozaktas and D. Mendlovic, "Fourier transforms of fractional order and their optical interpretation," Opt. Commun., vol. 101, no. 3-4, pp. 163-169, 1993.

[18] H. M. Ozaktas and D. Mendlovic, "Fractional Fourier optics," J. Opt. Soc. Amer. A, vol. 12, no. 4, pp. 743-751, 1995.

[19] L. B. Almeida, "The fractional Fourier transform and time-frequency representations," IEEE Trans. Signal Process., vol. 42, no. 11, pp. 3084-3091, Nov. 1994

[20] İ. Ş. Yetik, M. A. Kutay, H. Özaktaş, and H. M. Ozaktas, "Continuous and discrete fractional Fourier domain decomposition," in Proc. IEEE Int. Conf. Acoustics, Speech, Signal Processing (ICASSP), 2000, pp. I:93-I:96.

[21] İ. S. Yetik, H. M. Ozaktas, B. Barshan, and L. Onural, "Perspective projections in the space-frequency plane and fractional Fourier transforms," J. Opt. Soc. Amer. A, vol. 17, no. 12, pp. 2382-2390, 2000.
[22] M. A. Kutay, H. Özaktaş, H. M. Ozaktas, and O. Arıkan, "The fractional Fourier domain decomposition," Signal Process., vol. 77, no. 1, pp. 105-109, 1999.

[23] Ç. Candan, M. A. Kutay, and H. M. Ozaktas, "The discrete fractional Fourier transform,” IEEE Trans. Signal Process., vol. 48, no. 5, pp. 1329-1337, May 2000

[24] H. M. Ozaktas and O. Aytür, "Fractional Fourier domains," Signal Process., vol. 46, no. 1, pp. 119-124, 1995.

[25] O. Aytür and H. M. Ozaktas, "Non-orthogonal domains in phase space of quantum optics and their relation to fractional Fourier transforms," Opt. Commun., vol. 120, no. 3-4, pp. 166-170, 1995.

[26] F. Hlawatsch and G. F. Boudreaux-Bartels, "Linear and quadratic timefrequency signal representations," IEEE Signal Process. Mag., vol. 9 , no. 2 , pp. 21-67, 1992 .

[27] L. Cohen, Time-Frequency Analysis. Englewood Cliffs, NJ: Prentice Hall, 1995.

[28] H. M. Ozaktas, B. Barshan, D. Mendlovic, and L. Onural, "Convolution, filtering, and multiplexing in fractional Fourier domains and their relation to chirp and wavelet transforms," J. Opt. Soc. Amer. A, vol. 11, no. 2, pp. 547-559, 1994.

[29] K. B. Wolf, Integral Transforms in Science and Engineering. New York: Plenum Press, 1979.

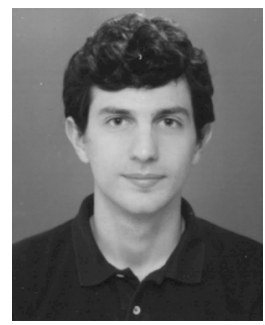

Haldun M. Ozaktas received the B.S. degree from Middle East Technical University, Ankara, Turkey, in 1987 and the Ph.D. degree from Stanford University, Stanford, CA, in 1991.

He joined Bilkent University, Ankara, Turkey, in 1991, where he is presently Professor of Electrical Engineering. In 1992, he was at the University of Erlangen-Nürnberg, Bavaria, as an Alexander von Humboldt Foundation Postdoctoral Fellow. In summer 1994, he worked as a Consultant at Bell Laboratories, Holmdel, NJ. He is the author of approximately 80 refereed journal articles, one book, many book chapters, and over 80 conference presentations and papers, over 25 of which have been invited. Four of his papers have been reprinted as milestone works, and a total of over 2000 citations to his work are recorded in the Science Citation Index (ISI). His academic interests include optical information processing, signal and image processing, and optoelectronic and optically interconnected computing systems.

Dr. Ozaktas is one of the youngest members of the Turkish Academy of Sciences and a Fellow of the Optical Society of America (OSA). He is the recipient of the 1998 ICO International Prize in Optics and one of the youngest recipients of the Scientific and Technical Research Council of Turkey Science Award (1999), among other awards and prizes.

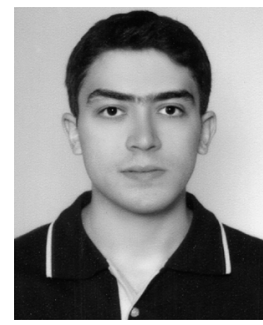

Uygar Sümbül (S'01) received the B.S. degree from Bilkent University, Ankara, Turkey, in 2003 and the M.S. degree from Stanford University, Stanford, CA, in 2005. He is currently working towards the Ph.D. degree at the Magnetic Resonance Systems Research Laboratory, Stanford University.

His current academic interests include magnetic resonance imaging, in particular high-temporal-resolution imaging, spectral analysis, and image processing.

Mr. Sümbül is a student member of the International Society for Magnetic Resonance in Medicine. 